\title{
Personalia
}

\section{Anatolij Svidzynskyj's 85th birthday}

This year a prominent Ukrainian theoretical physicist and public leader Anatolij Svidzynskyj marks his 85th birthday. Anatolij Svidzynskyj was born on 1 March 1929 in Mohyliv-Podilskyy (Ukraine) to a family of a physics teacher and an economist. Since 1939 and to the end of WWII Svidzynskyj's family lived in Kiev. In 1945 they moved to Lviv, where A. Svidzynskyj graduated from the Faculty of Physics and Mathematics of the Lviv State University. Vasyl Milyanchuk was his first supervisor. Due to his advice A. Svidzynskyj moved to Moscow State University where Mykola Bogolyubov worked at the time. Under Bogolyubov's supervision the PhD thesis "On the method of functional averaging in the theory of Green's function” was written, which was defended in 1956 at Lviv State University. One of the most important results obtained in the thesis is an exact solution of Bloch-Nordsieck model that describes an infrared asymptotic of the electron Green's function in a realistic theory -

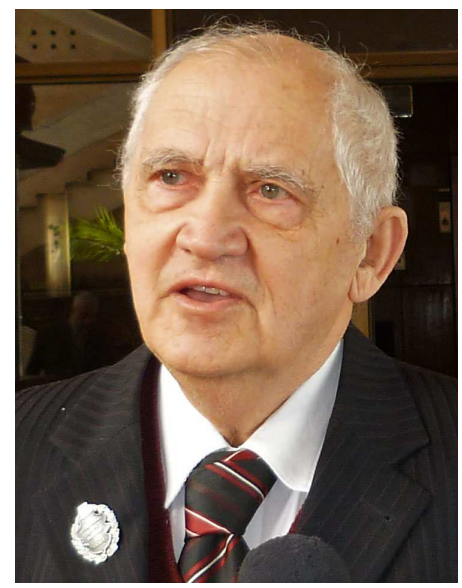
quantum electrodynamics. In this way, a non-analytical dependence of this function on a coupling constant was obtained, and the nature of infrared divergences in quantum electrodynamics was clarified.

Having defended his PhD thesis, A. Svidzynskyj received a position of an assistant in Kharkiv Polytechnic Institute at the Chair of Mathematical Physics and soon moved to a newly created academic Institute for Low Temperature Physics and Engineering. His work in this institute was very fruitful. In 1972 he defended his D.Sc. thesis on "Current states in spatially inhomogeneous superconducting systems". Among its important results is the application of the $T$-exponent parameterization based on a Poisson's formula for construction of a functional integral of the partition function of a superconductor. This led to a new compact formulation of the microscopic theory of superconductivity, which made it possible to move beyond the framework of a traditional method of generalized self-consistent field. Another result of this work consisted in solving the equations for the theory of superconductivity in a local approximation and in designing the equations for magnetic hydrodynamics of superconductors. In order to construct the microscopic theory of Josefson's effect, A. Svidzynskyi together with V. Slusarev developed a new and powerful method of the superconductivity equation averaging over atomic distances, which under the name of quasi-classical method is used by different authors up till now. Another important result, received in the doctoral thesis, was a theory of superconductive contacts. In particular, he succeeded in receiving a solution for current states in the superconductor-normal metal-superconductor contact.

In 1975 A. Svidzynskyj moved to the Simferopol State University, where he created a Chair for Theoretical Physics and became its unchangeable head. Over the years of his work in the Crimea, he trained plenty of young theoretical physicists. During this period, A. Svidzynskyj developed new methods in the theory of superconductor contacts, received a general formula for current in the systems of weak superconductivity, as well as developed a theory of superconductor contacts in the vicinity of critical temperature.

In 1993 following a suggestion by I. Yukhnovskyi, at that time the First Vice-Premier of Ukraine, A. Svidzynskyj moved to Lutsk to organize a classical university on the base of the Lutsk Lesya Ukrainka Pedagogical Institute. During 1993-1995 he worked as rector of the Volyn State University, where he created a Chair of Theoretical and Mathematical Physics, whose staff consists mainly of his former students that graduated from this university. Here he also wrote many textbooks and monographs, in particular, the textbook "Mathematical Methods of Theoretical Physics”, "Lectures on Thermodynamics”, "Lectures 
on High-temperature Superconductivity”, monograph “Microscopic Theory of Superconductivity” in two volumes.

A. Svidzynskyj has demonstrated a wide range of scientific interests. In the late 80 s, during the discussion regarding the creation of the Crimean Nuclear Power Plant, he analysed a number of issues connected with nuclear energetics, in particular, made a reasonable rating of the posterior probability of various types of accidents. Applying synergetic ideas he also investigated cultural problems that bring this branch of science to a completely new level. Among his scientific interests one can also find the theory of religion, political science, ethnology, philosophical problems of natural sciences and history.

Professor A. Svidzynskyj was trained in various physical and mathematical scientific schools Mykola Bogolyubov's, Lviv and Kharkiv, finally, he created his own - Anatolij Svidzynskyj's school, which was initiated and developed in different regions of Ukraine: in Sloboda Ukraine, in the Crimea and in Volyn.

On his $85^{\text {th }}$ birthday, editors of "Condensed Matter Physics" heartily congratulate Professor A. Svidzynskyj and wish him to stay in good health and new creative achievements. 